\title{
Use of a 4-D Cardiac Phantom to Quantify Karhunen-Loeve Images Applied to Myocardial Gated SPECT
}

\author{
L Comas, P Berthout, R Sabbah, JP Daspet, O Blagosklonov, \\ M Baud, J Verdenet, JC Cardot
}

\author{
Department of nuclear cardiology, Jean Minjoz Hospital, Besancon, France
}

\begin{abstract}
Myocardial gated single photon computed tomography (gSPECT) is widely used for quantification of myocardial perfusion and contractile function. Using a modified $4 D$ NCAT phantom, the purpose of this study was to evaluate the diagnostic performance of the Karhunen-Loeve transform (KLT) applied to gSPECT. For this purpose, perfusion was quantified by an index proportional to the size and the intensity of the defect $(\mathrm{Pi})$ and kinetics by an index (Ki) which was the mean endocardial and epicardial wall motion. KLT provides an uncorrelated image series in which only the first two (KLO,KL1) contain the main part of information. It was found that $K L O$ reflects perfusion and KLI kinetics.
\end{abstract}

\section{Introduction}

Cardiologists assume that analysis of the perfusion and motion of the heart, especially the left ventricle (LV), can give precise information about the health of the myocardium. With nuclear myocardial scan, it is possible to get sequences of images over the whole cardiac cycle; such sequences are real 3D movies of the cardiac motion [1]. Traditionally, assessment of regional function is made by expert visual evaluation but quantifying the degree and extent of the LV functional abnormalities permits a systematic assessment of the disease process on the myocardial performance.

The Karhunen-Loeve transform is well known for its compression and signal decorrelation properties. We use it routinely in planar gated blood pool studies. The goal of this study was to investigate KL transform for its capacity to quantify perfusion and wall motion in gSPECT.

Because of the difficulty in determining the true values in clinical studies, the analysis was performed using simulations with a phantom, so this approach allowed us to control the data and also to know the "truth". All simulated data for this study were generated using the 4-dimensional (4D) NURBS cardiac torso phantom (4D-NCAT) developed at the University of North Carolina at Chapel Hill [2]. The 4D NCAT phantom was developed to provide a realistic and flexible model of the human anatomy and physiology to be used in medical imaging research [3].

\section{Methods}

\subsection{Simulations}

The heart was generated by using a volume of 256 images of $256 * 256$ pixels. The field of view was $40 \mathrm{~cm}$. The cardiac cycle duration was 60 beats per minute and 8 volumes per cycle are obtained. Acquisition was simulated in ventral position. The patient was a man (without breast attenuation). The energy of the radio nuclide was $140 \mathrm{KeV}$. The number of counts per pixel was defined in the following way: $L V=100, V D=40$, Other tissues $=30$.

The heart was isolated in a $64 * 64$ pixels*64 slices zone (without interpolation) for each image cycle.

The resulting transaxial image sets were reoriented into short-axis, vertical and horizontal long-axis sets. From these series, we chose 3 short-axis slices (apical, mid and basal) and a mid-vertical long-axis slice to use a standard 20-segment classification (figure1).

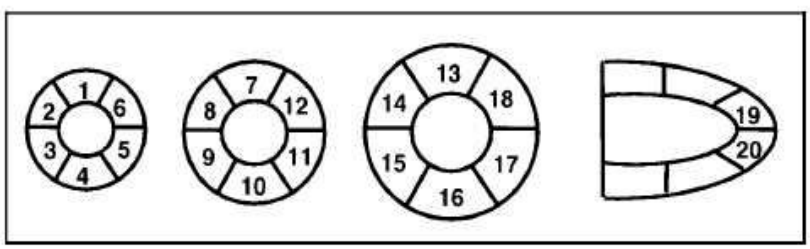

Figure 1. Segmentation in 20 sectors.

A lesion is defined in NCAT as an independent volume which is subtracted from normal thoracic volume. Three localizations of lesion were proposed corresponding to main coronary artery territories (anterior, lateral, inferior).

Perfusion defect : From a NCAT heart lesion volume, we determined a percentage of fixation in the lesion. Thus, we defined a population including one normal and 7 intensity reductions (10-20-30-40-50-60-70\%) applied in each of 3 territories (22 explorations).

Kinetics defect: We wanted to simulate the human 
heart as well as possible when it has kinetics defects. The diagram (figure 2) represents the functioning of a pathological heart in the inferior territory. It should be noted that endocardic displacement is always higher than epicardic displacement. The wall motion is generally lowered to the level of the hypo perfusion and dyskinetic zones. So, we transformed a heart lesion volume by modifying its time activity curve in 3 different ways before adding to the healthy heart.

Kinetics and perfusion defect: The two types of defects were cumulated to obtain a population of 55 explorations ( 1 normal, 3 territories $* 3$ time-activity curve modifications $* 6$ defect severities). We ignored the $10 \%$ defect severities because it did not appear on the images.

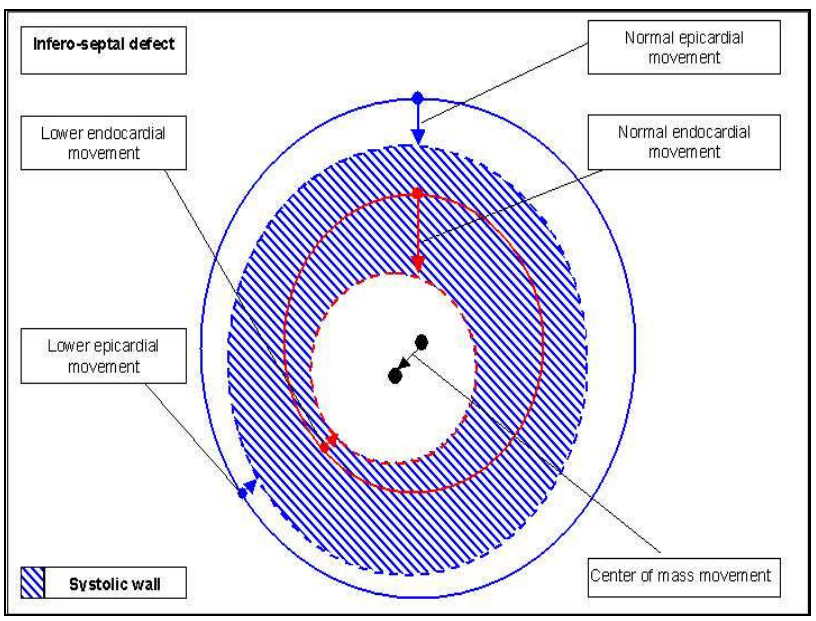

Figure 2. Schematization of the movements of the cardiac muscle

To be closer to the acquisitions carried out on the patient, it was necessary to take account, on the one hand, of the heart dimensions and, on the other hand, of the statistical noise.

Wall thickness : By 4 successive dilations, the cardiac wall was expanded. The intensity in each dilation zone corresponded to a decreasing percentage of the myocardium activity. The lesions, which underwent the same processing, were added to the heart and adjusted inside the cardiac wall in order to attenuate the abrupt transition between the lesion edge and the healthy wall.

Noise : it was generated by addition of a random gaussian type noise according to the following equation:

$$
R E S=A \pm 2 \times \sqrt{A} \times C
$$

Where $\mathrm{A}$ was the initial image and $\mathrm{C}$ a random matrix.

Finally, to maintain a continuity from one slice to another a 3D Butterworth smoothing (cut off frequency 0.75 , order 5) was applied to the whole rebuilt volume.

\subsection{Karhunen-Loeve transform}

The KLT is a change of space [4]; the new base orthogonal, is uncorrelated and ranked by decreasing order of information. Each pixel vector characterized by 8 components depending on time (very correlated in the base time) will be characterized by 8 components which are uncorrelated in the new base. The new axes are the eigen vectors of the correlation matrix. They permit to separate, as well as possible, the various temporal behaviours. The projection of the series on these new axes gives 8 principal images in this uncorrelated base. An compression effect can be obtained with a known loss because the new axes are ordered by decreasing value of information in the statistical meaning [5]. In the case of a synchronized cardiac exploration, $99 \%$ of information is contained in the first 3 principal images (KL0, KL1, KL2). KL0 represents the average image of the cardiac cycle. KL1 gathers the pixels by family of temporal evolution. At each end of the color scale, are the pixels whose temporal evolution is opposite and the background noise is around zero [6-7].

\subsection{Contours detection}

An automatic method of detection of contours [8], which we developed and validated, was applied to KL0 image of the various models. KL0 contours were used for KL1 image. For the segmentation in 20 angular sectors, the center was the center of mass of the myocardium.

\subsection{Perfusion and kinetics quantification}

For the quantification, we defined two indexes.

Perfusion: We calculated a perfusion index (Pi) characterizing the intensity and the extent of the lesion :

$$
\mathrm{Pi}=\mathrm{Nbt} \times 100 \times \mathrm{Al}
$$

Where Nbt was the total number of pixels of the myocardium, 100 corresponded to the intensity in the healthy myocardium and $\mathrm{Al}$ intensity was calculated directly in lesion volume. An identical calculation was carried out on each of the 20 sectors.

Kinetics: Motion index $\mathrm{Ki}$ was calculated in the following way:

$$
K i=\text { mean }((R p D-R p S)+(R n D-R n S)) / 2
$$

where $\mathrm{RpD}$ and $\mathrm{RpS}$ were epicardic diastolic and systolic radii and $\mathrm{RnD}$ and $\mathrm{RnS}$ were endocardic ones.

These two parameters were compared to QGS software where ejection fraction (EF), end-diastolique volume (EDV), end-systole volume (ESV), end-diastolique and end-systolique perfusion (ED and ES), motion and thickening are calculated automatically [9-10]. 


\section{Results}

An example is shown in figure 3

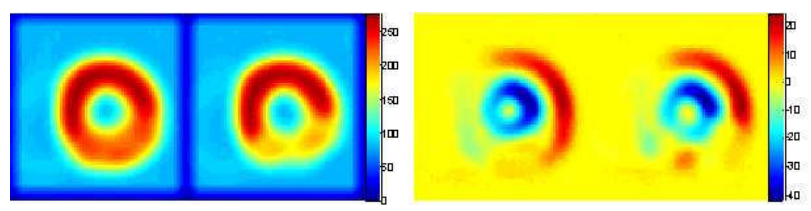

Figure 3 : KL0 and KL1 images for a normal model and a $40 \%$ perfusion and kinetics defect in an inferior territory.

The studied population comprised three kinds of models. For the perfusion analysis we had 22 explorations, for the kinetic defect analysis 10 and for the kinetics and perfusion defect analysis 55 explorations. For KL0 and KL1 images, the selected parameters were the minimum, maximum, mean and standard deviation. This analysis related to the whole image, the myocardial region of interest (LV ROI) and each left ventricle sector.

\subsection{Parameters validation}

In order to ensure the accuracy of the anomalies brought to the model and the suggested indices, method QGS was chosen and applied to explorations with perfusion and kinetics defect while taking for parameter $\mathrm{Ki}$ and $\mathrm{Pi}$ their 20 sectors mean value. We noted $0.28<\mathrm{EF}<0.42, \quad 38<\mathrm{EDV}<42 \mathrm{ml}, \quad 23<\mathrm{ESV}<30 \mathrm{ml}$, $2.39<$ motion $<3.71 \mathrm{~mm}$ and $12.72<$ thickening $<20.5 \%$, As with a usual patient population, motion and thickening were correlated with EF (0.96 and 0.97) and with ESV ( $\mathrm{r}=-0.84$ and -0.89 ).

Perfusion: Pi was compared to the ED and ES parameters. Pi was correlated with ED ( $\mathrm{r}=0.87)$ and with ES ( $\mathrm{r}=0.88)$.

Kinetics: Ki was compared to the EF, EDV and ESV and motion parameters. In order to take in account the different processing methods, we calculated the correlation between $\mathrm{Ki}$ and QGS parameters on 3 populations. Firstly, the 10 kinetic defect models with a not closed cavity apex slice were used. Ki was correlated to $\mathrm{EF}(\mathrm{r}=0.95)$ and to $\mathrm{ESV}(\mathrm{r}=-0.89)$. Secondly the 55 perfusion and kinetics defect explorations (where contours for the apex short-axis slice could have a closed cavity) were studied. Parameters had a poor correlation. Thirdly, only mid and basal short-axis slices were kept. In this case, correlation between $\mathrm{Ki}$ and $\mathrm{EF}$ was increased $(r=0.59$ to 0.79$)$ and also between $\mathrm{Ki}$ and motion $(r=0.61$ to 0.81 ).

The modifications brought to the time-activity curve on the lesion allowed the generation of various kinetics for which the parameters evolved like a true population. The defined parameters $\mathrm{Pi}$ and $\mathrm{Ki}$ were correlated with those routinely used by QGS.

\subsection{Perfusion defect}

For this analysis we used 22 models.

Whole image analysis : Only the KLO mean was correlated with $\mathrm{Pi}(\mathrm{r}=0.835)$ and even more so if we considered each axis separately.

LV ROI analysis : $\mathrm{KLO}_{\text {min }}$ and $\mathrm{KLO}_{\text {mean }}$ were correlated with $\mathrm{Pi}(\mathrm{r}=0.82$ and 0.95$)$. It should be noted that there was no correlation between the parameters of KL0 and those of KL1 and no correlation between Pi and KL1.

Sectorial analysis : it was carried out on 440 observations (183 healthy and 257 pathological). All the KL0 parameters were correlated with $\mathrm{Pi}$ whereas KL1 ones were independent (table 1).

Table 1. Correlation coefficients between KLO and Pi with 440 observations about perfusion defect.

\begin{tabular}{|c|c|c|c|c|}
\hline & $\mathrm{KL0}_{\min }$ & $\mathrm{KL0}_{\max }$ & $\mathrm{KL}_{\text {mean }}$ & $\mathrm{KL0}_{\text {sd }}$ \\
\hline $\mathrm{Pi}$ & 0.84 & 0.86 & 0.98 & 0.84 \\
\hline
\end{tabular}

Only KL0 image was sensitive to a perfusion defect.

\subsection{Kinetics defect}

For this analysis we used 10 models.

Whole image analysis : It should be noted that $\mathrm{KLO}_{\text {mean }}$ and $\mathrm{KLO}_{\text {sd }}$ were constant. No KLO parameter nor KL1 parameter was correlated with the parameters EF, EDV and ESV.

LV ROI analysis : The KL0 parameters were constant, whereas $\mathrm{KL}_{\text {min }}$ and $\mathrm{KL}_{\text {max }}$ varied but they were not correlated with the EF, EDV and ESV references.

Sectorial analysis : it was carried out on 200 observations. The KL0 parameters did not vary. Only the KL1 parameters were correlated with $\mathrm{Ki}$ (table 2). We observed no correlation between KL0 and KL1.

Table 2. Correlation coefficients between KL1 and $\mathrm{Ki}$ with 200 observations for kinetic defect

\begin{tabular}{|c|c|c|c|}
\hline & $\mathrm{KL}_{\text {min }}$ & $\mathrm{KL}_{\max }$ & $\mathrm{KL}_{\text {sd }}$ \\
\hline $\mathrm{Ki}$ & -0.85 & 0.82 & 0.90 \\
\hline
\end{tabular}

Only image KL1 was sensitive to the anomalies of kinetics expressed by the motion index.

\subsection{Perfusion and kinetics defect}

Perfusion and kinetics simultaneous anomaly corresponds to the most current situation.

For this analysis, we had 55 explorations.

Whole image analysis : Only, $\mathrm{KLO}_{\text {mean }}$ and $\mathrm{KLO}_{\text {sd }}$ was correlated with $\mathrm{Pi}(\mathrm{r}=0.80$ and 0.82$)$. No parameter of KL0 nor of KL1 was correlated with the EF, EDV and ESV parameters, although $\mathrm{KL}_{1} 1_{\text {min }}$ and $\mathrm{KL}_{1} 1_{\text {max }}$ varied.

$\mathrm{LV}$ ROI analysis : $\mathrm{KLO}_{\text {min }}$ and $\mathrm{KLO}_{\text {mean }}$ were correlated with $\mathrm{Pi}(\mathrm{r}=0.80$ and 0.91$)$, we noted no 
correlation between $\mathrm{Ki}$ and $\mathrm{KL}$ images.

Sectorial analysis : it concerned 1100 observations.

KL0 parameters were correlated with $\mathrm{Pi}$, KL1 parameters were correlated with Ki (tables 3-4).

Table 3. Correlation coefficients between KL0 and Pi with 1100 observations for perfusion and kinetics defect.

\begin{tabular}{|c|c|c|c|}
\hline & $\mathrm{KL0}_{\max }$ & $\mathrm{KLO}_{\text {mean }}$ & $\mathrm{KL0}_{\text {sd }}$ \\
\hline $\mathrm{Pi}$ & 0.88 & 0.90 & 0.92 \\
\hline
\end{tabular}

Table 4. Correlation coefficients between KL1 and $\mathrm{Ki}$ with 1100 observations for perfusion and kinetics defect.

\begin{tabular}{|c|c|c|c|}
\hline & $\mathrm{KL}_{\min }$ & $\mathrm{KL}_{\max }$ & $\mathrm{KL}_{\text {sd }}$ \\
\hline $\mathrm{Ki}$ & -0.63 & 0.75 & 0.76 \\
\hline
\end{tabular}

We noted no correlation between KL0 and KL1.

KL0 image reflected the perfusion and KL1 image reflected the wall motion.

\section{Discussion and conclusions}

The modifications model validation and the proposed perfusion and kinetics parameters were evaluated with QGS software which is commonly used in hospital routine. The apparent differences between our method and the QGS one came from various causes : The used slices automatic choice was not always the same from one method to another. It was more sensitive for the apical short-axis slice. The contours detection methods were different because of the intensity threshold (possible closed cavity for apical short-axis slice) and because the choice of the selected center of mass was not the same (seen on mid vertical long axis slice). Indeed, if we eliminated the apex short-axis and the long axis slices, we found a better correlation between $\mathrm{Ki}$ and $\mathrm{EF}$ and $\mathrm{ESV}$ $(r=0.59$ became 0.79 and -0.43 became -0.65$)$. This study showed that the modified NCAT model seemed well adapted to the measurement of perfusion and kinetic defects. However, it does not take account of the effect of partial volume since the images of diastole and systole have the same intensity and it is not possible to calculate a thickening parameter.

For KL quantitative analysis, we never observed correlation between KLO and KL1 images. From the 3step analysis (whole image, LV ROI, sector), we noted that it was not necessary to measure the parameters on the whole image, more especially in clinical routine where digestive artifact fixation often appears as important as the cardiac one. When LV ROI was observed, since the myocardium is never completely diseased, the healthy zones involved limits of parameters close to the normal. With the regional analysis, we showed that the $\mathrm{KLO}_{\text {mean }}$ and $\mathrm{KLO}_{\text {sd }}$ parameters were correlated with $\mathrm{Pi}$ and could be retained for a perfusion defect measurement; for the analysis of kinetics, since $\mathrm{KL} 1_{\text {mean }}$ was always close to 0 ,
$\mathrm{KL} 1_{\text {min }}, \mathrm{KL}_{\text {max }}$ and $\mathrm{KL} 1_{\text {sd }}$ were correlated with $\mathrm{Ki}$ and could be indicative for graduating the anomaly level.

The Karhunen-Loeve transform applied to myocardial gated SPECT was validated to the NCAT modified model Its interest was to show how to quantify the perfusion and kinetics anomalies with only two images, KL0 for perfusion and KL1 for kinetics. We are using this method on patients acquisitions which confirms these results.

\section{References}

[1] Paul AK and Nabi HA. Gated myocardial perfusion SPECT: basic principles, technical aspects, and clinical applications. J Nucl Med Technol, 2004;32(4):179-187.

[2] Segars WP. Development and Application of the new dynamic nurbs-based cardiac-torso (NCAT) phantom [dissertation]. Chapel Hill: North Carolina Univ.; 2001.

[3] Faber T, Modersitzki J, Folks RD and Garcia EV. Detecting changes in serial myocardial perfusion spect: A simulation study. J Nucl Cardiol, 2005;12:302-310.

[4] Fukunaga K. Introduction to Statistical Pattern Recognition. Boston: Academic Press; 1990.

[5] Narayanan MV, King MA, Wernick MN, Byrne CL, Soares EJ and Pretorius PH. Improved image quality and computation reduction in 4-D reconstruction of cardiacgated SPECT images. IEEE Trans Med Imaging, 2000;19(5):423-33.

[6] Blagosklonov O, Sabbah A, Verdenet J, and Cardot JC. Application of karhunen-loeve transform in nuclear cardiology: Spatio-temporal smoothing and quantitative image analysis. Computer in Cardiology, 2000;27:299302.

[7] Blagosklonov O, Berthout P, Comas L, Sabbah R, Verdenet J, Baud $\mathrm{M}$, and al. Using of karhunen-loeve transform for analysis of cardiac function in myocardial gated SPECT. SPIE Medical Imaging, 2003;5032:12171224 ,

[8] Comas L, Berthout P, Sabbah R, Blagosklonov O, Verdenet J, Baud M, and al. Automatic contours detection in myocardial gSPECT. Computer in Cardiology. 2003;30:621-624.

[9] Germano G, Kiat H, Kavanagh PB, Moriel M,. Mazzanti $\mathrm{M}, \mathrm{Su} \mathrm{HT}$, and al. Automatic quantification of ejection fraction from gated myocardial perfusion SPECT. J Nucl Med. 1995;36(11):2138-47.

[10] Germano G, Erel J, Lewin H, Kavanagh PB, and Berman DS. Automatic quantification of regional myocardial wall motion and thickening from gated technetium-99m sestamibi myocardial perfusion single-photon emission computed tomography. J Am Coll Cardiol. 1997;30(5):1360-7.

Address for correspondence

Laurent COMAS

Explorations cardiaques radio-isotopiques, Hôpital J. Minjoz 25030 Besancon Cedex, France

E-mail : lcomas@chu-besancon.fr 